\title{
Lessons from the cardiac arrhythmia suppression trial
}

\section{Deaths increased in patients given encainide or flecainide}

The cardiac arrhythmia suppression trial (CAST) was designed in the United States to test the hypothesis that suppressing asymptomatic ventricular premature beats with antiarrhythmic drugs reduces the risk of death from arrhythmia after myocardial infarction. The three drugs studied (encainide, flecainide, and moricizine) were chosen because they had already been shown to suppress ventricular premature beats in patients who had had infarcts.

Patients were entered into the trial between six days and two years after a myocardial infarction. To enrol patients at sufficiently high risk to ensure a clear result only those with impaired ventricular function were selected: they had to have an ejection fraction lower than 55\% if enrolled fewer than 90 days after their infarct or lower than $\mathbf{4 0 \%}$ if enrolled later. In the first part of the study the ability of the antiarrhythmic drugs to suppress ventricular premature beats was evaluated in individual patients. Once a drug was found that suppressed $80 \%$ of ventricular premature beats the patient was randomised to receive either that drug or placebo. After 1727 patients had been randomised the data were reviewed and the encainide and flecainide arms of the study were halted. Total mortality with treatment with encainide or flecainide had been higher than with placebo--7.7\% compared with $3 \cdot 0 \%$ (relative risk $2 \cdot 5,95 \%$ confidence interval 1.6 to $4 \cdot 5$ ). 2 The increased risk existed across all of the subgroups reported by the investigators.

The precise causes of the deaths associated with encainide and flecainide are not known. Both "death from arrhythmia" and death from non-arrhythmic cardiac disease were more common in the treated than the placebo group. Death from arrhythmia was defined as witnessed, instantaneous death without severe congestive heart failure or shock, unwitnessed death with no preceding change in symptoms and for which no other cause could be ascribed, or cardiac arrest.

Many of these deaths seem likely to have been due to an arrhythmogenic effect of encainide and flecainide. Arrhythmogenic effects of antiarrhythmic drugs are well known and may contribute directly to $5 \%$ of cases of cardiac arrest outside hospital. ${ }^{3}$ Life threatening arrhythmogenic effects have been reported with encainide and flecainide most often in patients with a history of sustained ventricular arrhythmias and very poor left ventricular function. ${ }^{+}$They have been reported only occasionally in patients with supraventricular arrhythmias and normal left ventricular function. ${ }^{5}$ Such effects usually occur shortly after the start of treatment or an increase in dosage. ${ }^{+}$None of the patients in the American trial, however, had a history of sustained ventricular arrhythmias, and active treatment was shown to increase the relative risk of death in patients with ejection fractions above as well as below $30 \%$. The excess rate of sudden death persisted throughout the 10 month follow up period, during which the drug dosage was unchanged. If the cause of the increased mortality was indeed provocation of arrhythmia then other factors, such as deteriorating ventricular function or worsening ischaemia, must have contributed.

Given that encainide and flecainide have been shown to be potentially lethal in this group of patients, what can be said about other antiarrhythmic drugs? The effect may be restricted to encainide and flecainide, to class lc agents, to all class 1 agents, or may apply to antiarrhythmic drugs in general. No other antiarrhythmic agents, however, have been evaluated in trials similar to the American trial. Disopyramide (a class la agent) has been shown not to reduce mortality in patients at relatively high risk when given for periods up to 14 days after myocardial infarction. ${ }^{6}$ Mexiletine (a class $1 \mathrm{~b}$ agent) has been shown to have no favourable effect on mortality after myocardial infarction in a small trial. ${ }^{7}$ In both of these studies drug treatment was associated with suppression of ventricular arrhythmias and a trend towards a worse prognosis. Placebo controlled trials of amiodarone after myocardial infarction are taking place, and the results are awaited. The CAST Data and Safety Monitoring Board has recommended that the study should continue with the third drug, moricizine, because no significant difference was found in mortality between patients treated with moricizine and placebo. Only 272 patients had been assigned to that arm of the trial, mainly because moricizine is relatively ineffective in suppressing ventricular premature beats.

What are the practical implications for the clinician? The main conclusion of the trial is that neither encainide nor flecainide should be used in patients with asymptomatic ventricular premature beats after myocardial infarction. In Britain such patients would not routinely be prescribed antiarrhythmic treatment, so that in this respect the study will have little effect on standard clinical practice. Encainide is not available in Britain. Flecainide was approved as second line treatment for patients with sustained junctional or ventricular arrhythmias that are resistant to conventional treatment or in whom conventional treatment is unsatisfactory. As an interim 
measure Riker $3 \mathrm{M}$ have now restricted this approval to patients with "life-threatening" arrhythmias only. There is no reason, however, why the original indications should be changed as a result of the trial; the relative risks and benefits of these and other antiarrhythmic drugs are not known in populations other than those studied in CAST. The manufacturers of encainide and flecainide might possibly discontinue production of the drugs as a result of this trial. This would be regrettable: these drugs provide the only effective treatment available for many patients with highly symptomatic or life threatening arrhythmias. Indeed, withdrawing treatment with the two drugs may already have led to the death of several patients.

The study has more general implications for the management of patients with arrhythmias. It has highlighted the risks associated with antiarrhythmic drugs in patients known to have had infarcts, and these risks may well apply to other antiarrhythmic drugs and to other forms of treatment. As a result of the trial non-pharmacological treatments for arrhythmias (such as antitachycardia pacemakers and catheter ablation) seem likely to be considered more readily, though these strategies, too, carry some risk.

The findings of the trial also have important implications for the design of future drug trials. Until now all trials of antiarrhythmic agents have been designed to compare efficacy with a placebo or another drug. In future, mortality should be given greater emphasis as a principal end point. This is not to say that the CAST study should be repeated with different drugs. The chances of showing overall benefit in terms of mortality are low in patients of this kind, and it would be unethical to treat patients without symptoms with the sole purpose of assessing the lethal potential of other agents. Patients with junctional arrhythmias are at even lower risk," and impossibly large numbers of patients would have to be enrolled to show any effect on mortality. One reasonable conclusion is that the monitoring of new antiarrhythmic agents in patients in this category should continue for longer periods after marketing to establish data on safety. In addition, "mortality trials" might be designed in patients known to be at a high risk of death, such as those with inducible or spontaneous sustained ventricular arrhythmias after myocardial infarction. Such trials should be performed in patients already fitted with implantable defibrillators so that otherwise life threatening arrhythmias could be identified and terminated. In this way the lethal potential of placebo or active drug could be investigated without loss of life.

The Cardiac Arrhythmia Suppression Trial is the first large long term trial that has attempted to assess whether antiarrhythmic drugs can reduce the risk of sudden death after myocardial infarction. It has highlighted the potential for harm associated with these agents; it has also raised many important questions about the development, assessment, and place of antiarrhythmic treatment.

CLIFFORD GARRATT Clinical Research Fellow

DAVID E WARD Consultant Cardiologist

A JOHN CAMM

Professor of Clinical Cardiology

Department of Cardiological Sciences,

St George's Hospital Medical School,

London SW 17 0RE

1 CAPS Investigators. The cardiac arrhsthmia pilot studv. Am 7 Curdiol 1986;57:91-5.

2 CAST Investigators. Preliminary report: effect of encainide and flecainide on mortalits in randomised trial of arrhythmia suppression after myocardial infarction. $N$ lingl $y$. Med 1989;321. 406-12.

3 Ruskin JN, Ml.Govern B, Garan H, DiMarco JP, Kelly E. Antiarrhythmic drugs: a possible cause of out-of-hospital cardiac arrest. N Engl f.lled 1983:309:1302-6.

+ Morganroth J. Risk factors for the development of proarrhythmic events. Am f Cardiol 1987:59: $32-7 \mathrm{E}$

Falk RH. Flecainide-induced ventricular tachycardia and fibrillation in patients treatced for atrial fibrillation. Ann Intern Med 1989;111:107-11.

6 UK Rythmodan Multicentre Study Group. Oral disopyramide after admission to hospital with suspected acute myocardial infarction. Postgrad Med f 1984;60:98-107.

Chamberlain DA, Jewitt DE, Julian DG, Campbel RWF, Boyle DM; Shanks RG. Oral mexiletine in high risk patients following myocardial infarction. Lancet 1980;ii: 1324-7.

8 Thumas GS. Death following withdrawal of encainide. $N$ kngl F Med 1989:321:393.

9 Anderson JL, Jolivette DM, Fredell PA. Summary of efficacy and safety of flecainide for supraventricular arrhythmias. Am 7 Ciardiol 1988;62:62-6D.

\section{Mothers with HIV}

\section{Risks to baby need to be balanced against benefits of breast feeding}

In New York last year AIDS was the most common cause of death in women aged between 25 and 35 (C Hankins, fifth international conference on AIDS, Montreal, 1989). In some parts of Rwanda as many as $30 \%$ of women aged between 26 and 40 are infected with HIV.' In Britain, by contrast, very few women are infected, but the number is growing and will determine the future incidence of paediatric cases. ${ }^{2}$ About a third of infants born to infected women are themselves infected; it has been estimated that by 1991 world wide most new cases of AIDS will be in children ( $J$ Chin, fifth international conference on AIDS, Montreal, 1989).

Most of the transmission from mother to child seems to occur in utero. ${ }^{3}$ But even a small risk of transmission by another route may be important in terms of absolute numbers. The main problem in estimating this risk comes from the current inability to determine whether a fetus is already infected at birth.

The contribution of breast feeding to neonatal infection is, therefore, hard to measure. HIV has been isolated from breast milk, ${ }^{+}$and several cases of infection attributed to breast feeding have been reported. ${ }^{56}$ In a series of 16 Zambian mothers who seroconverted after delivery three of their babies became infected, presumably through breast feeding ( $S$ Hira et $a l$, fifth international conference on AIDS, Montreal, 1989). In all these reports the mothers had had negative test results for HIV at delivery and probably became infected later. The likelihood of transmission shortly after infection may be especially high because there is a peak of viraemia at this time. The infectivity of breast milk may, therefore, depend partly on the mother's stage of infection.

Most mothers with HIV infection are, however, already infected at the time of delivery. Their risk of subsequently transmitting HIV in breast milk is less easy to assess. Several studies have compared the prevalence of HIV infection in breast fed versus non-breast fed infants, finding little-or no difference between the two groups (C Hutto et al, fifth international conference on AIDS, Montreal, 1989). ${ }^{7}$ One explanation of this is that a woman who does not transmit HIV in utero has a low infectivity and is also unlikely to transmit HIV in her milk. 